\title{
Ship Detection With TanDEM-X Data Extending the Polarimetric Notch Filter
}

\author{
Armando Marino, Member, IEEE, and Irena Hajnsek, Fellow, IEEE
}

\begin{abstract}
Synthetic aperture radar plays a vital role in ship detection due to the possibility of acquiring high-resolution images at nighttime and under cloud cover. This letter is focused on improving ship detection, exploiting the capability of TanDEM-X to collect interferometric data. Currently, along-track interferometry is used to estimate the speed of ocean surface currents or vessels. The detection of ships plays an important role in the retrieval of vessel speed and is mostly executed exploiting only one of the TanDEM-X images (i.e., not taking advantage of the availability of a second interferometric image). The aim of this study is to extend the capabilities of a ship detector previously developed by the authors, namely, geometrical perturbation-polarimetric notch filter (GP-PNF), to include single-pass interferometric information acquired by TanDEM-X. Interestingly, such enhancement makes it possible to employ the GP-PNF with single-polarization data as well. The proposed algorithms and their statistical behavior are tested on five Tandem-X dual-polarimetric HH/VV scenes acquired in the North Sea. The detection results are validated, exploiting the Automatic Identification System location of vessels. All of the new GP-PNF versions show good performance and provide larger vessel-sea contrast compared with single-channel detectors.
\end{abstract}

Index Terms-Interferometry, polarimetry, ship detection, synthetic aperture radar (SAR), TanDEM-X.

\section{INTRODUCTION}

$\mathbf{T}$ HE main feature of ships in synthetic aperture radar (SAR) images is a relatively large backscatter signal. This is why several ship detectors perform a statistical test on the intensity of a single SAR image [1]-[5]. Apart from detection, ship classification and delineation is a topic of large interest [6].

Polarimetry can improve detection, since it provides more independent measurements [7]. Furthermore, the polarimetric behavior of the sea can be generally singled out (e.g., a rough surface modeled by Bragg scattering [8]). Several approaches were proposed [1], [9]-[13], and in this work, the geometrical perturbation-polarimetric notch filter (GP-PNF) [11], [14]-[17] will be analyzed.

Manuscript received December 22, 2014; revised February 27, 2015, May 8, 2015, June 11, 2015, and June 30, 2015; accepted July 1, 2015. Date of publication July 24, 2015; date of current version August 7, 2015. This work was supported by the HGF Alliance "Remote Sensing and Earth System Dynamics" through Project HA310.

A. Marino is with the Department of Engineering and Innovation, Open University, Milton Keynes MK7 6AA, U.K. (e-mail: armando.marino@open. ac.uk).

I. Hajnsek is with the Institute of Environmental Engineering, ETH Zurich, 8092 Zurich, Switzerland, and also with the Microwaves and Radar Institute, German Aerospace Center (DLR), 82234 Wessling, Germany.

Color versions of one or more of the figures in this paper are available online at http://ieeexplore.ieee.org.

Digital Object Identifier 10.1109/LGRS.2015.2453235
The aim of this letter is to enhance the GP-PNF exploiting the single-pass interferometric information acquired by TanDEM-X. Along-track interferometry has been largely employed to measure the speed of surface currents [18], [19] or vessels [20]. The detection of ships plays an important role in the processing chain that delivers the vessel speed. This letter proposes two new versions of the GP-PNF, and one of them is exploiting a single-polarimetric mode (single-pol). Dualpolarimetric data are not routinely acquired for maritime applications, and a solution that considers single-pol is appealing for operational purposes. Remarkably, it is the first time that the GP-PNF is applied on single-pol data.

A quad-pol SAR system can acquire four polarization channels that can be arranged in a scattering vector $\underline{k}_{L}=$ $[H H, H V, V H, V V]^{T}$, where $H$ and $V$ stand for linear horizontal and vertical, and the repeated letter is for transmitterreceiver [7], [21]. In the case of a reciprocal medium and a monostatic sensor, $H V=V H$, and $\underline{k}$ is three-dimensional complex. In this letter, distributed targets are characterized by their second-order statistics exploiting the target covariance matrix $[C]=\left\langle\underline{k} \underline{k}^{* T}\right\rangle$, where $\langle$.$\rangle is the finite averaging operator.$

\section{GP-PNF}

The GP-PNF was derived starting from a more general methodology aimed at processing multidimensional images. That methodology was defined as the geometrical perturbation filter (GPF) [22]-[25]. The GPF considers a perturbed version of the target to detect, and then, it checks for coherence between original and perturbed targets in the data. A feature partial scattering vector is introduced [25], i.e.,

$\underline{t}=\left[\left\langle\left|k_{1}\right|^{2}\right\rangle,\left\langle\left|k_{2}\right|^{2}\right\rangle,\left\langle\left|k_{3}\right|^{2}\right\rangle,\left\langle k_{1}^{*} k_{2}\right\rangle,\left\langle k_{1}^{*} k_{3}\right\rangle,\left\langle k_{2}^{*} k_{3}\right\rangle\right]^{T}$

where $k_{i}$ 's are the components of the scattering vector.

The GP-PNF modifies the concept of polarimetric perturbation analysis inverting the detection role, to suppress the return from a selected target [14], [15], [26], [27]. The polarimetric behavior of the sea clutter can be identified by a single feature vector $\underline{t}_{\text {sea }}$. On the other hand, ships can have a large variety of polarimetric signatures depending on orientation, material, and structures [6]. To bypass this issue, the GP-PNF focuses on targets lying in the complementary orthogonal subset of the sea vector, i.e.,

$$
\gamma_{n}=\left(1+\frac{R e d R}{\underline{t}^{* T} \underline{t}-\left|\underline{t}^{* T} \hat{\underline{t}}_{\mathrm{sea}}\right|^{2}}\right)^{-\frac{1}{2}} .
$$

The RedR is a scalar detector parameter. $\underline{\underline{t}}_{\text {sea }}$ is the normalized version of $\underline{t}_{\text {sea }}$ that is extracted using a large window defined 


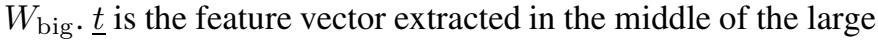
window exploiting a smaller window $W_{\text {small }}$.

\section{A. Extending the GP-PNF to Interferometric Data}

The mathematical formulation presented in (2) is flexible and can be adapted for different typologies of multidimensional images (i.e., different observables could be included in the feature vector). With TanDEM-X, it is possible to build polarimetric and interferometric (Pol-InSAR) covariance matrices [28]. If dual-pol HH/VV data are considered, the Pol-InSAR scattering vector is $\underline{k}=\left[H H_{1}, V V_{1}, H H_{2}, V V_{2}\right]^{T}$, and the Pol-InSAR covariance matrix can be estimated as: $\left[C_{4}\right]=\left\langle\underline{k k^{* T}}\right\rangle$. Following this reasoning, different elements of $\left[C_{4}\right]$ can be included in the feature vector.

- The original version of the GP-PNF employs $\left\langle\left|H H_{1}\right|^{2}\right\rangle$, $\left\langle\left|V V_{1}\right|^{2}\right\rangle$, and $\left\langle H H_{1} V V_{1}^{*}\right\rangle$ [17] that will be used in this letter as well. $\left\langle\left|H H_{2}\right|^{2}\right\rangle,\left\langle\left|V V_{2}\right|^{2}\right\rangle$, and $\left\langle H H_{2} V V_{2}^{*}\right\rangle$ are not supposed to add extra information (as long as the bistatic angle is not very large).

- $\left\langle H H_{1} H H_{2}^{*}\right\rangle$ and $\left\langle V V_{1} V V_{2}^{*}\right\rangle$ contain the interferometric information. Due to the along-track baseline (ATB) and the very short decorrelation time of the sea, these elements are supposed to be very small for the sea clutter. In particular, it was shown that an ATB larger than $60 \mathrm{~m}$ is sufficient to strongly decorrelate the sea [19]. On the other hand, vessels are supposed to maintain correlation, unless the baselines are very large (e.g., several kilometers) [20]. Furthermore, since the interferometric phase is always a mix of cross- and along-track components, vessels and sea are supposed to present different behaviors due to differences in their height or speed. Summarizing, both the magnitude and phase of these elements are expected to expose differences between sea and vessels. As a final remark, if the ATB is smaller than $60 \mathrm{~m}$ or larger than several kilometers, these terms should not provide significant improvement to the ordinary GP-PNF, since vessels and sea will present very similar behaviors.

- Cross elements as $\left\langle H H_{1} V V_{2}^{*}\right\rangle$ contain a mixture of polarimetric and interferometric information that is not of trivial interpretation. Moreover, they should not add information that is not already enclosed in the previous elements.

Interestingly, the exploitation of an ATB was already found beneficial in [29], where COSMO Sky-Med data in dualpolarimetric ping-pong mode were used, exploiting the fact that the $H H$ and $V V$ channels are separated by an ATB.

The new feature vectors are built as follows:

$$
\begin{aligned}
\underline{t}_{n}= & {\left[\left\langle\left|H H_{1}\right|^{2}\right\rangle,\left\langle\left|V V_{1}\right|^{2}\right\rangle,\left\langle H H_{1} V V_{1}^{*}\right\rangle\right]^{T} } \\
\underline{t}_{\text {full }}= & {\left[\left\langle\left|H H_{1}\right|^{2}\right\rangle,\left\langle\left|V V_{1}\right|^{2}\right\rangle,\left\langle H H_{1} V V_{1}^{*}\right\rangle,\left\langle H H_{1} H H_{2}^{*}\right\rangle\right.} \\
& \left.\times\left\langle V V_{1} V V_{2}^{*}\right\rangle\right]^{T} \\
\underline{t}_{H H}= & {\left[\left\langle\left|H H_{1}\right|^{2}\right\rangle,\left\langle H H_{1} H H_{2}^{*}\right\rangle\right]^{T} } \\
\underline{t}_{V V}= & {\left[\left\langle\left|V V_{1}\right|^{2}\right\rangle,\left\langle V V_{1} V V_{2}^{*}\right\rangle\right]^{T} . }
\end{aligned}
$$

When the GP-PNF is exploited with $\underline{t}_{H H}$ or $\underline{t}_{V V}$, only singlepol data are required; hence, the acronym PNF may be misleading, and they will be referred to as GP-NF. In the following, the outputs of the new detectors will be named distances, and the symbols $\gamma_{n}, \gamma_{\text {full }}, \gamma_{H H}$, and $\gamma_{V V}$ will be used.

\section{Statistical Distribution of Distances}

The derivation of the GP-PNF probability density function (pdf) is presented in [30]. Here, only the final expression is reported, considering a generic GP-PNF distance represented by the symbol $\gamma_{x}$, i.e.,

$$
\begin{aligned}
f_{\Gamma_{x}}\left(\gamma_{x} \mid H_{0}\right)= & \frac{2}{\Gamma(N)}\left(\frac{N}{\mu}\right)^{N}\left(\operatorname{RedR} \frac{\gamma_{x}^{2}}{1-\gamma_{x}^{2}}\right)^{N+1} \frac{2}{\operatorname{RedR}} \gamma_{x}^{-3} \\
& \times \exp \left[-\operatorname{RedR} \frac{N}{\mu} \frac{\gamma_{x}^{2}}{1-\gamma_{x}^{2}}\right] \operatorname{rect}\left[\gamma_{x}-\frac{1}{2}\right]
\end{aligned}
$$

where $N$ is the equivalent number of looks, $\mu$ is the mean of the target power expressed as $P_{t}=\underline{t}^{* T} \underline{t}-\left|\underline{t}^{* T} \underline{\hat{t}}_{\text {sea }}\right|^{2}, \operatorname{Red} R$ is a detector parameter, and $\Gamma(N)$ is the $\Gamma$ function of argument $N$. $H_{0}$ stands for null hypothesis (only clutter), in contrast with $H_{1}$ that represents the alternative hypothesis (target). Since the procedure to derive the pdf is the same, independently of the content of the feature vector, (4) should theoretically fit all the distances proposed. This assumption is tested in the following.

The pdf parameters $\mu$ and $N$ can be estimated on a ring around a test area that is protected by a guard window [30]. $\operatorname{RedR}$ is a free detector parameter and is chosen to have a pdf with mean 0.15 . While the second-order statistics can be estimated using large windows $W_{\mathrm{big}}$, the pdf parameters should be estimated exploiting guard windows, since a target in the scene may contaminate the estimation of pdf parameters. These issues are more thoroughly discussed in [30].

The statistical test exploited in this letter is based on the Neyman-Pearson lemma for the likelihood ratio (LR) [17], i.e.,

$$
\Lambda=\frac{f_{\Gamma}\left(\gamma_{n} \mid H_{1}\right)}{f_{\Gamma}\left(\gamma_{n} \mid H_{0}\right)}=\frac{\operatorname{rect}\left[\frac{\gamma_{n}-\left(\gamma_{n}^{\min }+1\right) / 2}{1-\gamma_{n}^{\min }}\right]}{f_{\Gamma}\left(\gamma_{n} \mid H_{0}\right)} .
$$

Currently, a very simple pdf for the vessels is considered, where valid targets are assumed to have minimum power $P_{t}^{\min }$ (that is then converted into $\gamma_{n}^{\mathrm{min}}$ ). A simple pdf keeps the treatment more general, but in the future, work will be carried out to include better vessel statistical models.

\section{Test With Real TanDEM-X Data}

\section{A. Presentation of the Data}

In this letter, six TanDEM-X HH/VV scenes are considered, which were acquired in the winter of 2012 in the North and Baltic Seas. A survey was carried out during the acquisitions collecting wind speed and Automatic Identification System (AIS) positions. For all the images, the azimuth resolution is $6.7 \mathrm{~m}$, whereas the slant range resolution is $1.1 \mathrm{~m}$ (Table I summarizes further details).

For the sake of brevity, figures are presented only for one scene; however, a complete analysis is reported at the end. The dual-pol Pauli RGB composite color image for the Boknafjorden scene is presented in Fig. 1(a). The color coding is as follows: Blue is the filtered intensity of \langle|$H H_{1}+$ $\left.\left.V V_{1}\right|^{2}\right\rangle$, red is the filtered intensity $\left\langle\left|H H_{1}-V V_{1}\right|^{2}\right\rangle$, and 
TABLE I

Details on HH/VV TANDEM-X IMAGES EXPLOITED IN THE COMPARISON. TIME IS IN UTC

\begin{tabular}{lcccccc}
\hline \hline Date & Location & $\begin{array}{c}\text { Incidence } \\
\text { angle }\end{array}$ & $\begin{array}{c}\text { Ground } \\
\text { range res. }\end{array}$ & $\begin{array}{c}\text { Wind speed } \\
\mathrm{m} / \mathrm{sec}\end{array}$ & $\begin{array}{c}\text { Ships } \\
\text { with AIS }\end{array}$ & $\begin{array}{c}\text { Along-track } \\
\text { baseline (m) }\end{array}$ \\
\hline \hline 03/12/2012(06:33) & Aberdeen (South) & $\sim 33.5^{0}$ & $2.1 \mathrm{~m}$ & $6.7(\mathrm{SE})$ & 11 & 294 \\
\hline $03 / 12 / 2012(06: 33)$ & Aberdeen (North) & $\sim 33.5^{0}$ & $2.1 \mathrm{~m}$ & $6.7(\mathrm{SE})$ & 7 & 294 \\
\hline $11 / 12 / 2012(16: 36)$ & Baltic (Bornholm) & $\sim 26^{0}$ & $2.6 \mathrm{~m}$ & $10.3(\mathrm{~N})$ & 5 & 378 \\
\hline $11 / 12 / 2012(16: 36)$ & Baltic (Binz) & $\sim 26^{0}$ & $2.6 \mathrm{~m}$ & $12.3(\mathrm{~N})$ & 6 & 378 \\
\hline $21 / 12 / 2012(06: 33)$ & Boknafjorden & $\sim 31.9^{0}$ & $2.1 \mathrm{~m}$ & 7.7 to $12.3(\mathrm{SE})$ & 7 & 292 \\
\hline $06 / 01 / 2013(17: 02)$ & Kristiansand & $\sim 34.9^{0}$ & $2.0 \mathrm{~m}$ & $5.1(\mathrm{~N})$ & 2 & 342 \\
\hline
\end{tabular}

green is the filtered magnitude of the copolarization cross term $\left\langle H H_{1} V V_{1}^{*}\right\rangle$.

Initially, it is valuable to evaluate if the interferometric correlation can be used to discriminate between sea and vessels. For this reason, Fig. 1(e) shows in red and blue the magnitude of $\left\langle H H_{1} H H_{2}^{*}\right\rangle$ and $\left\langle V V_{1} V V_{2}^{*}\right\rangle$. Interestingly, the sea presents a lower correlation compared with vessels or land. Similar results were observed over all the scenes. However, since some vessel may show decorrelation, the authors would not suggest using the correlation on its own.

\section{B. GP-PNF Distances}

Fig. 1(b)-(d) presents the GP-PNF distances. The small window employed by the GP-PNF is $W_{\text {small }}=33 \times 33$ pixels, whereas the large window is $W_{\mathrm{big}}=151 \times 151$ pixels. $R e d R$ is selected to locate the distributions around 0.15 . It is interesting to observe that these distances make distinguishing sea clutter from vessels easy.

\section{Statistical Behavior}

Here, two main points will be investigated: Is the analytical pdf derived in [17] and [30] fitting the new distances? Which distance has the lowest statistical variation? Fig. 1(i)-(n) presents the histograms of the different distances (circles: data histograms; solid line: fitted pdf). The area used to calculate the histograms is represented inside a red rectangle in the RGB image. The theoretical pdf seems able to characterize the statistical behavior of the distances. Additionally, comparing the different versions, it is possible to observe that the variances of $\gamma_{n}, \gamma_{H H}$, and $\gamma_{V V}$ are comparable, whereas $\gamma_{\text {full }}$ presents a slightly smaller variance (which is a desired property). To obtain some quantitative result, the two-sample Kolmogorov-Smirnov and the $\chi^{2}$ tests are performed. All the distributions passed the tests exploiting 50 samples.

\section{Detection Performance}

The LR is exploited for detection. The pdf parameters are estimated using a guard window of $151 \times 151$ pixels around the central pixel and a ring window that extends to $201 \times$ 201 pixels. The parameters used for the NP test are $\alpha=0.9$ and $P_{t}^{\min }=3 \cdot 10^{-4}$. Fig. $1(\mathrm{f})$ and $(\mathrm{g})$ presents the detection masks for $\gamma_{\text {full }}$ and $\gamma_{H H}$ (the other masks are omitted because they are very similar to these two). It can be observed that all the vessels and islands are detected with complete absence of false alarms, although $\gamma_{\text {full }}$ detects one azimuth ambiguity. Azimuth ambiguities can be screened out in pre- or postprocessing [31]. Finally, several points over islands and the mainland are detected (they can be screened out using a land mask).
To have a larger comparison, constant false-alarm rate (CFAR) tests on the intensity of the $H H$ and $V V$ channels are considered. The pdf employed is the K-distribution, and the threshold is selected locally (as for the GP-PNF) solving the integral numerically. The result for the $H H$ channel is presented in Fig. 1(h) (the $V V$ channel provides similar results). Again, the same targets identified by the GP-PNF were detected by the CFAR, showing comparable performances on this dataset.

Table II summarizes the results over all the scenes. Some comments are provided in the following.

Probability of Detection $P_{d}$ : All the detectors are able to identify the vessels with AIS, showing comparable performance in terms of $P_{d}$. Only one target is missing from the single-channel detection, and this is a buoys $(2 \times 2 \mathrm{~m})$ in the scene Aberdeen North.

Probability of False Alarm $P_{f}$ : Here, the comparison shows different performances. The GP-PNF detectors do not present any false alarms at the exception of azimuth ambiguities, whereas there are scenes where the single-channel CFAR is largely affected by false alarms. More specifically, in the Baltic scenes, the sea has a relatively low backscattering with very large heterogeneity due to convection cells and rolls. The false alarms in Kristiansand are due to bright swells (having large backscattering, they are detected by the CFAR). The GP-PNF detectors are more robust against these false alarms, because they are based on the polarimetric or temporal behavior of the clutter (rough surface), which is relatively unaffected by its brightness. As a final remark, it is important to keep in mind that the exploitation of morphological filters may improve the results for the Baltic scenes, where false alarms appear mostly as isolated points. This is at the expense of eventually losing some small targets.

Mean Contrast Between Vessels and Sea: The contrast can be used as a measure of separability between targets and clutter. Here, it is calculated as the mean of the detector distance for ship pixels divided by the mean for sea pixels (land areas are masked out): $c=\left\langle\operatorname{dist}\left(x_{\text {ship }}\right)\right\rangle /\left\langle\operatorname{dist}\left(x_{\text {sea }}\right)\right\rangle$, where $x$ represents a generic pixel, and dist is the considered distance (or parameter). The contrast is averaged to provide some information that is not related to one single ship.

To have a fair comparison, for the GP-PNF, we calculated the contrast using the root square of the target power $\operatorname{sqrt}\left(P_{t}\right)$. This is linearly proportional to the channel intensity and represents the backscattering that is associated to a target that is orthogonal to the sea in the feature vector space. The contrast is not calculated using the distances $\gamma_{x}$, since they are dimensionally different from an intensity (they are normalized), and they depend on the choice of RedR. The mean contrast is presented in the lower line of Table II. The contrast for the $\mathrm{HH}$ and VV intensities is calculated following two strategies: exploiting 


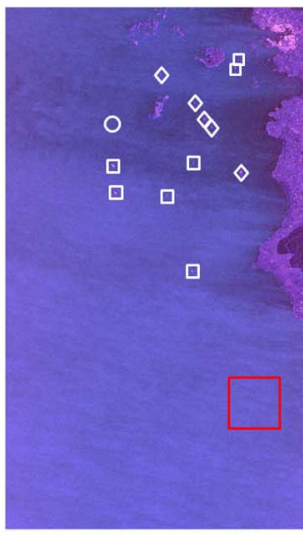

(a)

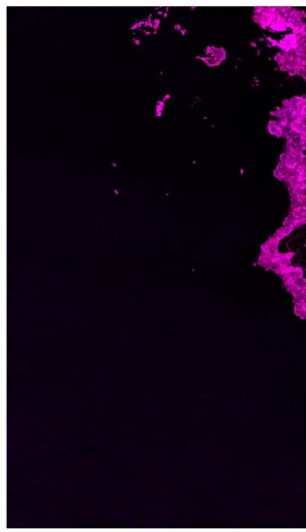

(e)

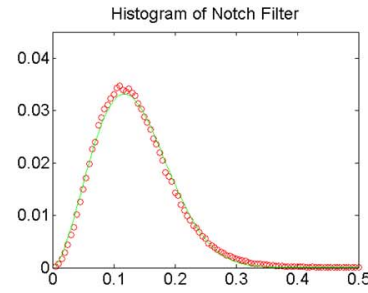

(i)

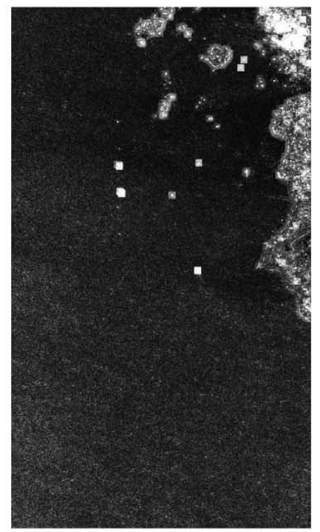

(b)

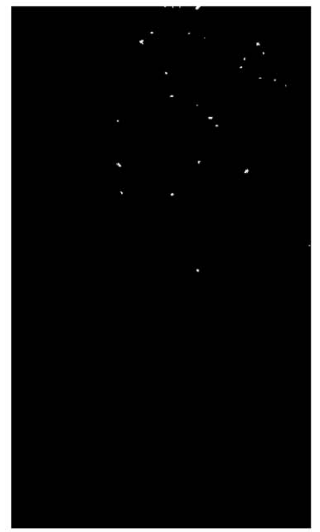

(f)

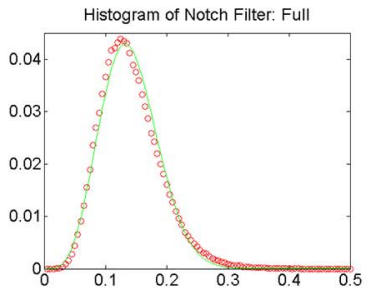

(j)

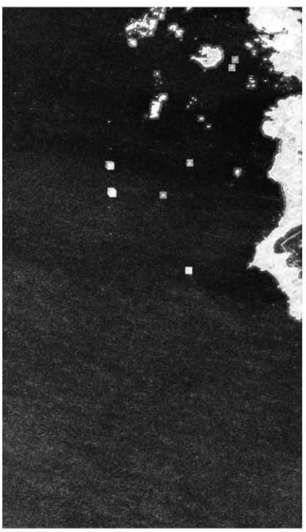

(c)

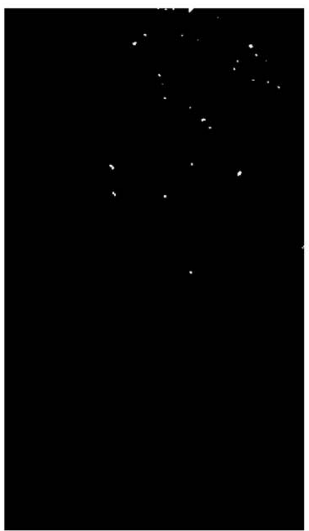

(g)

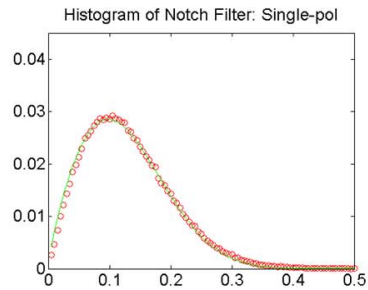

(k)

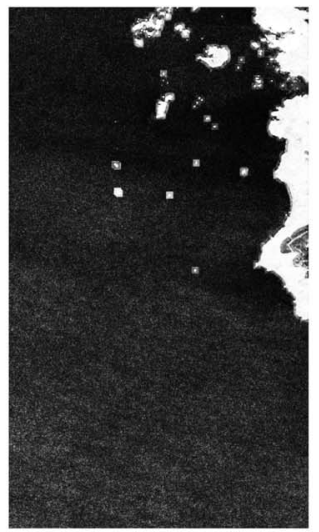

(d)

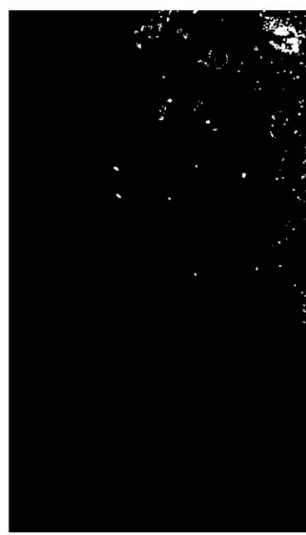

(h)

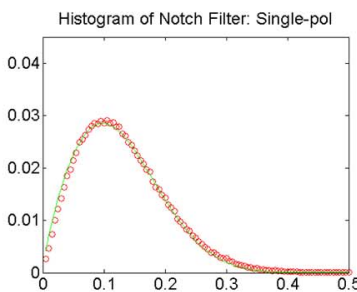

(1)

Fig. 1. TanDEM-X, Boknafjorden (Norway), 21/12/2012. (a) RGB Pauli color composite image. Red: $|H H-V V|^{2}$; green: $\left|H H \cdot V V^{*}\right|$; blue: $\mid H H+$ $\left.V V\right|^{2}$. Rectangles: vessels; diamonds: islands; circle: azimuth ambiguity; red rectangle: area for training. The GP-PNF distances are linearly scaled between zero and one. (b) $\gamma_{n}$. (c) $\gamma_{\text {full }}$. (d) $\gamma_{H H}$. (e) RGB of cross correlation between interferometric channels. Red: $H H$ channel; green: zero; blue: $V V$ channel. (f) and (g) Consider a Neyman-Pearson on the LR: $\alpha=0.9$ and $P_{t}^{\min }=2 \cdot 10^{-4}$ on $\gamma_{\mathrm{full}}$ and $\gamma_{H H}$, respectively. (h) Detection mask with a K-distribution on the intensity of the $H H$ channel, $P_{f}=10^{-6}$. (i)-(n) Histograms of different versions of the notch filter: (i) $\gamma_{n}$; (l) $\gamma_{\mathrm{full}}$; (m) $\gamma_{H H}$; (n) $\gamma_{V V}$.

a box car filter of size $W_{\text {small }}$ and not performing any filtering (showed in brackets). The three $P_{t}$ distances have higher contrast compared with $\mathrm{HH}$ and VV intensities. This is because the notch filter strongly reduces the polarimetrically homogeneous areas and enhances the areas presenting polarimetric heterogeneity. Comparing the different distances, it can be observed that we cannot easily identify the detector that always produces the best contrast. For this reason, we may conclude that $\gamma_{\text {full }}$ may be preferred, considering that it has the distribution with lower variance.

\section{DISCUSSIONS AND CONCLUSIONS}

In this letter, an extension of the GP-PNF ship detector has been proposed to exploit the capability of TanDEM-X to acquire polarimetric and interferometric data. The selection of the detector strongly depends on the main task for which the data are acquired, but some general suggestions may be given.

Single-Polarimetric Data for Along-Track Studies: The exploitation of a GP-NF provides much better contrast than the intensity of a single channel, and therefore, it may be suggested to use it. It is also to be said that if we are interested in detecting medium-to-large vessels, then the ordinary CFAR on a single intensity may be sufficient. However, the latter will need to be accompanied by a powerful methodology to remove false alarms in postprocessing.

Dual-Polarimetric Data for Along-Track Studies: Considering the better contrast and higher rejection of false alarms, we would recommended to exploit the extra information provided by polarimetry using $\gamma_{n}$ or $\gamma_{\text {full }}$. The latter may be preferred since it provides a smaller variance.

Ship Detection Only: If the purpose of the analysis is exclusively ship detection (without any analysis of the vessel speed), 
TABLE II

Detection Results. Up: Number of Vessel Detected/Number of False Alarms/Azimuth Ambiguities; Down: Mean Contrast. The Numbers in Bracket Present the Mean Contrast When Filtering Is Not Performed (I.e., Single Look)

\begin{tabular}{ccccccc}
\hline \hline Detector & Aberdeen South & Aberdeen North & Baltic North & Baltic South & Boknafjorden & Kristiansand \\
\hline \hline \multirow{2}{*}{ HH intensity } & $11 / 0 / 6$ & $6 / 0 / 2$ & $5 /$ thousands $/ 0$ & $6 /$ tens/0 & $7 / 0 / 0$ & $2 /$ tens/0 \\
\hline \multirow{2}{*}{ VV intensity } & $17.09(16.99)$ & $31.91(40.28)$ & $8.87(10.87)$ & $6.65(4.63)$ & $8.47(7.87)$ & $8.57(8.91)$ \\
\hline & $11 / 0 / 5$ & $6 / 0 / 2$ & $5 /$ thousands/0 & $6 /$ tens/0 & $7 / 0 / 0$ & $2 /$ tens/0 \\
$\gamma_{n}$ & $11 / 0 / 14$ & $25.82(27.72)$ & $8.78(12.63)$ & $5.64(3.43)$ & $5.52(4.87)$ & $8.91(8.04)$ \\
\hline & 66.60 & $7 / 0 / 5$ & $5 / 0 / 0$ & $6 / 0 / 2$ & $7 / 0 / 3$ & $2 / 0 / 0$ \\
$\gamma_{f u l l}$ & $11 / 0 / 15$ & 54.01 & 72.50 & 54.94 & 44.79 & 65.52 \\
\hline & 65.34 & $570 / 7$ & $5 / 0 / 1$ & $6 / 0 / 0$ & $7 / 0 / 1$ & $2 / 0 / 0$ \\
$\gamma_{H H}$ & $11 / 0 / 12$ & $7 / 0 / 3$ & $5 / 0 / 1$ & $6 / 0 / 0$ & $7 / 0 / 0$ & $2 / 0 / 0$ \\
\hline & 64.92 & 60.68 & 45.22 & 52.85 & 36.93 & 50.86 \\
$\gamma_{V V}$ & $11 / 0 / 10$ & $7 / 0 / 3$ & $5 / 0 / 1$ & $6 / 0 / 0$ & $7 / 0 / 0$ & $2 / 0 / 0$ \\
\hline
\end{tabular}

the exploitation of TerraSAR-X dual-polarimetric data may be sufficient. Therefore, we would probably not suggest acquiring TanDEM-X data if the speed of the vessels is not of interest.

One remarkable finding is that single-polarimetric TanDEM-X data can also be used to largely improve the contrast provided by the intensity detector. Therefore, if TanDEM-X data are available, it is worthwhile to take advantage of the interferometric capability for the detection as well.

\section{ACKNOWLEDGMENT}

The authors would like to thank DLR, in the framework of the proposal POLIN_1734, for providing TanDEM-X data.

\section{REFERENCES}

[1] D. J. Crisp, "The state-of-the-art in ship detection in synthetic aperture radar imagery," Dept. Def., Australian Gov., Canberra, Australia, 2004.

[2] K. Eldhuset, "An automatic ship and ship wake detection system for spaceborne SAR images in coastal regions," IEEE Trans. Geosci. Remote Sens., vol. 34, no. 4, pp. 1010-1019, Jul. 1996.

[3] P. W. Vachon, "Ship detection in synthetic aperture radar imagery," in Proc. OceanSAR, St. John's, NL, Canada, Oct. 2006, pp. 1-5.

[4] S. Brusch et al., "Ship surveillance with TerraSAR-X," IEEE Geosci. Remote Sens. Lett., vol. 9, no. 3, pp. 1092-1103, Mar. 2011.

[5] P. Iervolino, R. Guida, and P. Whittaker, "NovaSAR-S and maritime surveillance," in Proc. IEEE IGARSS, Melbourne, Vic., Australia, Jul. 2013, pp. 1-4.

[6] G. Margarit, J. J. Mallorquí, J. M. Rius, and J. Sanz-Marcos, “On the usage of GRECOSAR, an orbital polarimetric SAR simulator of complex targets for vessel classification studies," IEEE Trans. Geosci. Remote Sens., vol. 44, no. 12, pp. 3517-3526, Dec. 2006.

[7] S. R. Cloude, Polarisation: Applications in Remote Sensing. Oxford, U.K.: Oxford Univ. Press, 2009.

[8] C. R. Jackson and J. R. Apel, Synthetic Aperture Radar Marine Users Manual, U.S. Dept. Commerce, Nat. Ocean. Atmos. Admin. (NOAA), Washington, DC, USA, 2004.

[9] C. Liu, P. W. Vachon, and G. W. Geling, "Improved ship detection using polarimetric SAR data," in Proc. IEEE IGARSS, Anchorage, AK, USA, Sep. 2004, pp. 1800-1803.

[10] F. Nunziata, M. Migliaccio, and C. E. Brown, "Reflection symmetry for polarimetric observation of man-made metallic targets at sea," IEEE J. Ocean. Eng., vol. 37, no. 3, pp. 384-394, Jul. 2012.

[11] D. Velotto, F. Nunziata, M. Migliaccio, and S. Lehner, "Dual-polarimetric TerraSAR-X SAR data for target at sea observation," IEEE Geosci. Remote Sens. Lett., vol. 10, no. 5, pp. 1114-1118, Sep. 2013.

[12] R. Touzi, "On the use of polarimetric SAR data for ship detection," in Proc. IEEE IGARSS, Hamburg, Germany, Jun. 1999, pp. 812-814.

[13] W. L. Cameron, N. N. Youssef, and L. K. Leung, "Simulated polarimetric signatures of primitive geometrical shapes," IEEE Trans. Geosci. Remote Sens., vol. 34, no. 3, pp. 793-803, May 1996.
[14] A. Marino, "A notch filter for ship detection with polarimetric SAR data," IEEE J. Sel. Topics Appl. Earth Observ. Remote Sens., vol. 6, no. 3, pp. 1219-1232, Jun. 2013.

[15] A. Marino, M. Sugimoto, K. Ouchi, and I. Hajnsek, "Validating a notch filter for detection of targets at sea with ALOS-PALSAR data: Tokyo Bay," IEEE J. Sel. Topics Appl. Earth Observ. Remote Sens., vol. 7, no. 12, pp. 4907-4918, Dec. 2014.

[16] A. Marino and N. Walker, "Ship detection in variable sea states and depolarised sea clutter: A polarimetric notch filter," in Proc. POLinSAR, Frascati, Italy, Jan. 2011, pp. 1-6.

[17] A. Marino and I. Hajnsek, "Ship detection with TanDEM-X data: A statistical test for a polarimetric notch filter," in Proc. EUSAR, Berlin, Germany, Jun. 2014, pp. 1-4.

[18] R. Romeiser et al., "Quality assessment of surface current fields from TerraSAR-X and TanDEM-X along-track interferometry and Doppler centroid analysis," in IEEE Trans. Geosci. Remote Sens., vol. 52, no. 5, pp. 2759-2772, May 2013.

[19] S. Suchandt, A. Lehmann, and H. Runge, "Analysis of ocean surface currents with TanDEM-X ATI: A case study in the Baltic sea," in Proc. IGARSS, Quebec City, QC, Canada, Jul. 2014, pp. 3918-3921.

[20] S. V. Baumgartner and G. Krieger, "Ship detection and motion parameter estimation with TanDEM-X in large along-track baseline configuration," in Proc. SEASAR, Tromso, Norway, Jun. 2012, pp. 1-4.

[21] J. S. Lee and E. Pottier, Polarimetric Radar Imaging: From Basics to applications. Boca Raton, FL, USA: CRC Press, 2009.

[22] A. Marino, S. R. Cloude, and I. H. Woodhouse, "A polarimetric target detector using the Huynen fork," IEEE Trans. Geosci. Remote Sens., vol. 48, no. 5, pp. 2357-2366, May 2010.

[23] A. Marino, A New Target Detector Based on Geometrical Perturbation Filters for Polarimetric Synthetic Aperture Radar (POL-SAR). New York, NY, USA: Springer-Verlag, 2012.

[24] A. Marino, S. R. Cloude, and J. M. Lopez-Sanchez, "A new polarimetric change detector in radar imagery," IEEE Trans. Geosci. Remote Sens., vol. 51, no. 5, pp. 2986-3000, May 2013.

[25] A. Marino, S. R. Cloude, and I. H. Woodhouse, "Detecting depolarized targets using a new geometrical perturbation filter," IEEE Trans. Geosci. Remote Sens., vol. 50, no. 10, pp. 3787-3799, Oct. 2012.

[26] A. Marino and N. Walker, "Ship detection with quad polarimetric TerraSAR-X data: An adaptive notch filter," Proc. IEEE IGARSS, Vancouver, BC, Canada, Jul. 2011, pp. 245-248.

[27] A. Marino, N. Walker, and I. Woodhouse, "Ship detection with RADARSAT-2 quad-pol SAR data using a notch filter based on perturbation analysis," in Proc. IEEE IGARSS, Honolulu, HI, USA, Jul. 2010, pp. 3704-3707.

[28] S. R. Cloude and K. P. Papathanassiou, "Polarimetric SAR interferometry," IEEE Trans. Geosci. Remote Sens., vol. 36, no. 5, pp. 1551-1565, Sep. 1998.

[29] F. Nunziata and M. Migliaccio, "On the COSMO-SkyMed pingpong mode to observe metallic targets at sea," IEEE J. Ocean. Eng., vol. 38, no. 1, pp. 71-79, Jan. 2013.

[30] A. Marino and I. Hajnsek, "Statistical tests for a ship detector based on the polarimetric notch filter," IEEE Trans. Geosci. Remote Sens., vol. 53, no. 8, pp. 4578-4595, Aug. 2015.

[31] A. Monti Guarnieri, "Adaptive removal of azimuth ambiguities in SAR images," IEEE Trans. Geosci. Remote Sens., vol. 43, no. 3, pp. 625-633, Mar. 2005. 